\title{
Analysis of Non-fortuitous Predictive States of the RC4 Keystream Generator ${ }^{\star}$
}

\author{
Souradyuti Paul and Bart Preneel \\ Katholieke Universiteit Leuven, Dept. ESAT/COSIC, \\ Kasteelpark Arenberg 10, \\ B-3001 Leuven-Heverlee, Belgium \\ \{Souradyuti.Paul, Bart.Preneel\}@esat.kuleuven.ac.be
}

\begin{abstract}
The RC4 stream cipher is the most widely used software based stream cipher. It is based on a secret internal state of $N=256$ bytes and two pointers. This paper proposes an efficient algorithm to compute a special set of RC4 states named non-fortuitous predictive states. These special states increase the probability to guess part of the internal state in a known plaintext attack and present a cryptanalytic weakness of RC4. The problem of designing a practical algorithm to compute them has been open since it was posed by Mantin and Shamir in 2001. We also formally prove a slightly corrected version of the conjecture by Mantin and Shamir of 2001 that, using only $a$ known elements along with the two pointers at some round, the RC4 pseudorandom generation algorithm cannot produce more than $a$ outputs in the next $N$ rounds.
\end{abstract}

\section{Introduction}

RC4 is the most widely used software based stream cipher. The cipher has been integrated into SSL and WEP implementations. RC4 is extremely fast and its design is simple. The cipher was designed by Ron Rivest in 1987 and kept as a trade secret until it was leaked out in 1994.

In this paper we formally prove the conjecture (due to Mantin and Shamir [1]) that only $a$ known elements along with $i$ and $j$ at any RC4 round cannot predict more than $a$ output bytes in the next $N$ rounds. The set of non-fortuitous predictive states reduces the data and time complexity of the branch and bound attack on RC4 [1,7]. So far there was no efficient algorithm to obtain those states. The main achievement of this paper is that we design a practical two-phase recursive algorithm to determine the non-fortuitous predictive states. The complexity is far less than the trivial exhaustive search for small values of $a$.

\subsection{Description of RC4}

RC4 runs in two phases (description in Fig. 1). The first part is the key scheduling algorithm KSA which takes an array $S$ to derive a permutation

\footnotetext{
* This work was partially supported by the Concerted Research Action GOAMEFISTO-666 of the Flemish government.
} 
of $\{0,1,2, \ldots, N-1\}$ using a variable size key $K$. The second part is the output generation part PRGA which produces pseudo-random bytes using the permutation derived from KSA. Each iteration or 'round' produces one output value. Plaintext bytes are bit-wise XOred with the output bytes to produce ciphertext. In most of the applications RC4 is used with word length $n=8$ bits and $N=256$. The symbol $l$ denotes the byte-length of the secret key.
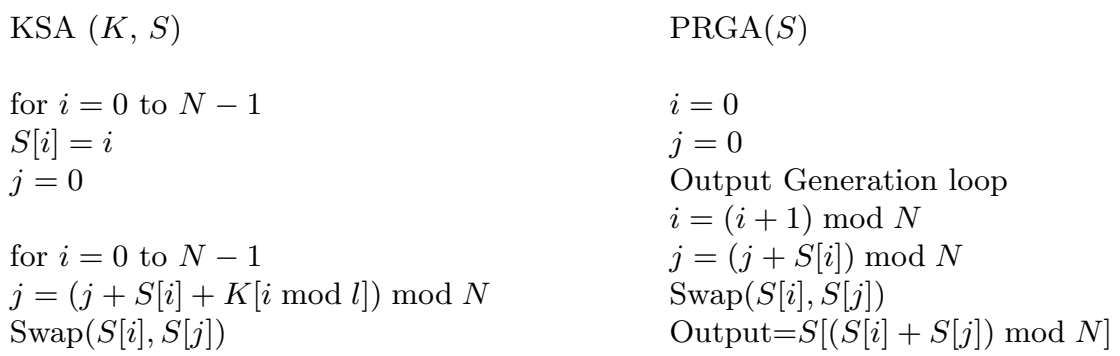

Fig. 1. The Key Scheduling Algorithm (KSA) and the Pseudo-Random Generation Algorithm (PRGA)

\subsection{Previous Attacks on RC4}

RC4 came under intensive scrutiny after it has been made public in 1994 . Finney showed in [3] a class of states that RC4 will never enter. The class contains all the states for which $j=i+1$ and $S[j]=1$. A fraction of approximately $N^{-2}$ of all possible states fall under Finney's forbidden states. It is simple to show that these states are connected by a cycle of length $N(N-1)$. We know that RC4 states are also connected in a cycle and the initial state, where $i=0$ and $j=0$, is not one of the Finney's forbidden states. Finney's forbidden states play a significant role in the analysis of non-fortuitous predictive states.

Jenkins detected in [5] a probabilistic correlation between the secret information $(S, j)$ and the public information ( $i$, output). Golić in [6] showed a positive correlation between the second binary derivative of the least significant bit output sequence and 1. Fluhrer and McGrew in [2] observed stronger correlations between consecutive bytes. Properties of the state transition graph of RC4 were analyzed by Mister and Tavares [9]. Grosul and Wallach demonstrated a related key attack that works better on very long keys. Andrew Roos also discovered in [10] classes of weak keys. Knudsen et al. have attacked versions of RC4 with $n<8$ by their backtracking algorithm in which the adversary guesses the internal state and checks if an anomaly occurs in later stage [7]. In the case of 
contradiction the algorithm backtracks through the internal states and re-guesses.

The most serious weakness in RC4 was observed by Mantin and Shamir in [1] where they found that the probability of occurrence of zero at the second round is twice as large as expected. In broadcast applications a practical ciphertext only attack can exploit this weakness.

Fluhrer et al. in [11] have recently shown that if some portion of the secret key is known then RC4 can be broken completely. This is of practical importance because in the Wired Equivalence Privacy Protocol (WEP in short) a fixed secret key is concatenated with IV modifiers to encrypt different messages. In [12] it is shown that the attack is feasible.

Mironov, in [4], modelled RC4 as a Markov chain and recommended to dump the initial $12 \times N$ bytes of the output stream (at least $3 \times N$ ) in order to obtain uniform distribution of the initial permutation of elements.

\subsection{Non-fortuitous Predictive RC4 States}

Definitions of Fortuitous state and Predictive State are given in [2] and [1] respectively. We restate the definition of a Fortuitous state.

Definition 1. The RC4 state (i.e., S-Box elements, $i$ and $j$ ), in which only $m$ consecutive $S$-Box elements are known and only those $m$ elements participate in producing the next $m$ successive outputs, is defined to be a fortuitous state of length $m$.

In [1] a set of special RC4 states, known as Predictive States, has been conceptualized. Below we give the definition of a Predictive State with a little modification to the one given in [1] to suit our analysis.

Definition 2. Let $A$ be an a-state (i.e., only a elements of the S-Box, $i$ and $j$ are known at some round which is defined to be round 0) which produces $b$ outputs (not necessarily consecutive) at rounds $1, r_{2}, r_{3}, \ldots$, $r_{b} \leq N$. Then $A$ is said to be b-predictive a-state.

A $b$-predictive $a$-state necessarily means that the execution of RC4 does not stop before $b$ known outputs are produced. The RC4 execution only stops when $i$ or $j$ is not available at some round, that is, when the internal state can not be updated deterministically at some round. In the definition, the first assertion is that an a-state is the snapshot of the RC4 state immediately before the first predicted output ( $a$ elements of the SBox that do not produce any output are of no importance in the present context). Secondly, we set the upper bound on $r_{b}$ to $N$ instead of $2 N$ as mentioned in [1]. 
It is clear from the above definitions that the set of a-predictive astates is a superset of the set of fortuitous states of length $a$ as any fortuitous state of length $a$ is clearly an a-predictive a-state. Below we give the definition of a non-fortuitous predictive state of length a.

Definition 3. If an $a$-predictive $a$-state is not $a$ fortuitous state of length $a$ then the state is a non-fortuitous predictive state of length $a$.

We apologize that the term "non-fortuitous" is a misnomer. The term was coined to contrast it with the fortuitous states defined in [2]. In fact, "non-fortuitous" predictive states are fortuitous too and are far more complex to derive. As the term non-fortuitous predictive state is too long, in the rest of the paper we will use non-fortuitous state synonymously with it.

\section{Importance of Predictive States}

As mentioned in [1], the existence of $b$-predictive $a$-states is important for the cryptanalyst as $a$ elements of the S-Box including $j$ (note that the $i$ value is always available to the cryptanalyst) can be extracted with non-trivial probability by observing $b$ specific output bytes in the output segment.

Let the events $E_{A}$ and $E_{B}$ denote the occurrences of an a-state and the corresponding $b$ outputs when the $i$ value of an a-state is known. We assume uniformity of the internal state and the corresponding external state for any fixed $i$ value of the internal state. Assuming $a$ much smaller than $N$ and disregarding the small bias induced in $E_{B}$ due to $E_{A}$, we apply Bayes' Rule to get,

$$
P\left[E_{A} \mid E_{B}\right]=\frac{P\left[E_{A}\right]}{P\left[E_{B}\right]} P\left[E_{B} \mid E_{A}\right] \approx \frac{N^{-(a+1)}}{N^{-b}} \cdot 1=N^{b-a-1}
$$

On the average one of the $N^{a+1-b}$ occurrences of the event $E_{B}$ is caused by the event $E_{A}$. From the above equation it is evident that a cryptanalyst will be interested in those b-predictive a-states for which $P\left[E_{A} \mid E_{B}\right]$ is maximum. In such case the number of false hits, for which the occurrences of $b$ specific words in the output stream (here denoted by the event $E_{B}$ ) are not induced by that specific internal state (here denoted by the event $E_{A}$ ), will be minimum. Maximizing $P\left[E_{A} \mid E_{B}\right]$ is equivalent to maximizing $b-a$ (see Eqn. (1)).

To determine the maximum value of $b$ for a given $a$, we first formally prove the very important theorem that if a $b$-predictive $a$-state exists then 
$a \geq b$. This was left as a conjecture in [1]. ${ }^{1}$ So, the best attack along this line is to obtain all the a-predictive a-states, keep the information a priori in a database indexed by the values of the $i$ pointer (sorted by outputs) and look up the output sequence for a possible match.

Throughout the paper $S_{r}[l]$ denotes the element of the S-Box indexed by $l$ at the $r^{\text {th }}$ round (whether $S_{r}[l]$ is before or after the swapping at the $r^{\text {th }}$ round, should be understood in the context); the first predicted output corresponds to round 1 . Similarly, $i_{t}$ and $j_{t}$ denote the values of $i$ and $j$ respectively at round $t$. Unless otherwise stated $S[i]$ and $S[j]$ denote the elements of the S-Box pointed to by $i$ and $j$ respectively at the round in question. All arithmetic operations are done modulo $N$.

Theorem 1. If any b-predictive a-state exists then $a \geq b$.

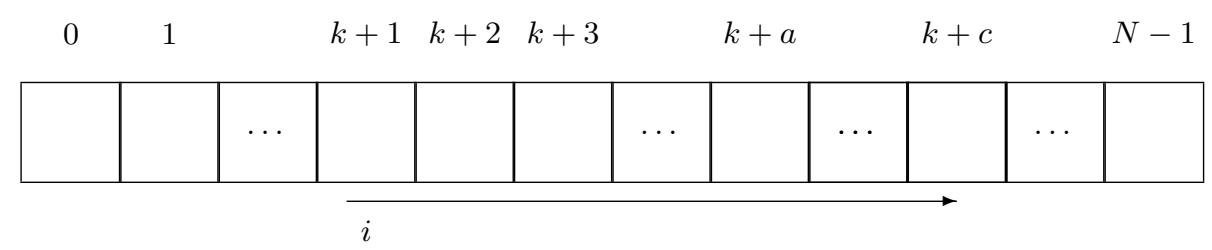

Fig. 2. Prediction of $b$ outputs in $c$ rounds where only $a$ elements are known in the S-Box at round 0.

Proof. The theorem is trivially true when $a=N$ as, according to definition, outputs are predicted in the next $N$ rounds only. Therefore, if $a=N$ elements of the S-box are known they will produce $b=N$ elements in the next $N$ rounds. Below we consider the case when $a<N$. Let us assume that an $a$-state produces $b$ outputs and $b>a$. As shown in Fig. 2, all the $b$ outputs are generated, as the $i$ pointer sweeps through all the positions from the index $k+1$ till the index $k+c$, in $c$ rounds $(b \leq c \leq N)$. The first and the last outputs are produced at the $(k+1)^{t h}$ and $(k+c)^{t h}$ rounds respectively. In each of the $c$ rounds $i, j$ should be available otherwise RC4 halts forever (see PRGA in Fig. 1) because the swapping operation cannot be executed deterministically.

Lemma 1. Starting from any a-state (i.e., at round 0) no unknown element of the S-box becomes known in the subsequent rounds of the execution of the pseudorandom generation algorithm of $R C 4$.

\footnotetext{
${ }^{1}$ In [1], the upper bound on $r_{b}$ (see Def. 2 and [1]) is given to be $2 N$. But within this bound the conjecture is not true, as we see that, for the trivial case of an $N$-state, $2 N$ outputs can be predicted in $2 N$ rounds.
} 
Proof (Lemma). Let $V=\left\{U_{1}, U_{2}, \cdots, U_{a}, K_{1}, \cdots, K_{N-a}\right\}$ denote the set of elements arranged in some order in the S-box where the $U_{i}$ 's are unknown elements and the $K_{j}$ 's are known elements at round 0 . Note that the subscripts associated with the elements (for example, $i$ of $U_{i}$ ) do not indicate their indices in the S-box. Observe that, with the execution of RC4 pseudorandom bit generator, only the positions (i.e., the indices) of the elements change. Suppose an unknown element (say $U_{l}$ ) at round 0 becomes known at some RC4 round $t$ where $t \geq 1$. This fact implies that there must be an operation to assign a value to $U_{l}$ during the execution. But the only transformation the RC4 pseudorandom generation algorithm performs is the transposition of elements of the S-box (see Fig. 1). Thus, we reach a contradiction, which proves the lemma.

Availability of $j$ at each of these rounds implies that $S[i]$ is also available because the previous value of $j$ is known (note, $j=j+S[i]$ ). From the above lemma, $S[i]$ is one of the known elements of the $a$-state in each of the $c$ rounds. In each of the output producing $b$ rounds a necessary condition is that $S[i]+S[j]$ is known. This fact along with the above lemma imply that $S[i]$ and $S[j]$ are individually known in each of those $b$ rounds. No empty cells ${ }^{2}$ in the S-Box can be filled with a known value in any of the output producing rounds as the swapping takes place between the known elements. As $i$ takes $c$ different values of index in $c$ rounds and every time $S[i]$ is known, we should have a maximum of $(c-b)$ swaps where $S[j]$ is unknown. In each of these $(c-b)$ swaps (where $S[i]$ is known but $S[j]$ is unknown) at most one new-cell ${ }^{3}$ will be filled with a known element. So, a maximum of $(c-b)$ different new-cells between $k+1$ and $k+c$ will be filled with known elements at least once in the aforementioned $c$ rounds. Consequently, a minimum of $b$ different indices in the S-Box between $k+1$ and $k+c$ must be occupied with as many known elements at round 0 , otherwise we reach a scenario where we have at least one new-cell which is never filled with a known elements in $c$ rounds. This is impossible as $S[i]$ is known in each of the $c$ rounds as a necessary condition. As we have only $a$ known elements where $a<b$, we reach a contradiction. Therefore, our assumption (i.e., $a<b$ ) is wrong. We conclude that $b \leq a$, i.e., an $a$-state can not predict more than $a$ outputs in the next $N$ rounds.

We have already defined an $a$-state as the partially specified state of RC4 (i.e., $a$ elements of S-Box, $i$ and $j$ ) just before the $1^{\text {st }}$ output is predicted.

\footnotetext{
${ }^{2}$ By an empty cell we denote an S-Box location with unknown element at the current round.

${ }^{3}$ A new-cell is an S-Box location with unknown element at round 0.
} 
We emphasize that $a$ elements of the S-Box that predict $b$ outputs may change its positions as RC4 runs. So taking the snapshot of the $a$ elements of the S-Box immediately before the round of the $1^{\text {st }}$ predicted output is cryptanalytically equivalent to those $a$ elements at some other rounds.

Corollary 1. Only a known elements of the $S$-Box along with two pointers at any $R C 4$ round cannot predict more than a outputs in the next $N$ rounds.

Proof. The proof is immediate from Theorem 1.

\subsection{Necessary and Sufficient Condition for a Predictive State to be Non-fortuitous}

Quite understandably, the larger the number of a-predictive a-states, the larger will be the probability to obtain one of them under the reasonable assumption of uniformity of RC4 states.

Fortuitous states can be easily obtained using the state counting algorithm described in [2]. Our objective is to develop a method to determine non-fortuitous states of any length. The following theorem and the corollary divide a-predictive a-states into fortuitous states and non-fortuitous states.

Theorem 2. An a-predictive $a$-state is a fortuitous state of length $a$ if and only if the predicted output words are consecutive.

Proof. The only if part of the theorem is direct from the definition of a fortuitous state. Now we prove the if part.

We prove it by contradiction. Let us assume that there exists at least one a-predictive a-state for which the outputs are consecutive but the elements of the S-Box are not consecutive. We assume that the generation of outputs starts when $i$ points to $S_{1}[k+1]$ (see Fig. 2) and the $a^{t h}$ output is issued when $i$ points to $S_{a}[k+a]$. During the passage of $i$ from the index $(k+1)$ to the index $(k+a)$, the number of outputs generated is also $a$. So, each of the $a$ rounds produces output. Let us assume that there is at least one empty cell, say $S_{0}[k+t]$, between the indices $k+1$ and $k+a$ at round 0 . At the $t^{t h}$ round, $S_{t}[k+t]$ should contain a known value otherwise $j$ cannot be updated at this round and consequently no output can be predicted. As $S_{0}[k+t]$ was initially empty, there is a round, say the $r^{t h}$ round where $r<t$, when one known value will be swapped into the empty $S_{r}[k+t]$ for the first time (note there may be many such rounds). As a consequence, the $r^{\text {th }}$ round does not produce output because swapping 
takes place when $j$ points to $S_{r}[k+t]$ which is unknown at that time. But we assumed that each of the $a$ rounds produces output. Eventually we reach a contradiction. Therefore, our assumption, i.e., the S-Box elements are not consecutive, is wrong.

As things stand, we have $a$ consecutive elements that are fixed in the S-Box and we get $a$ outputs in the next $a$ rounds. This is just the case of a fortuitous state of length $a$.

Corollary 2. An a-predictive $a$-state is a non-fortuitous state of length $a$ if and only if the predicted output words are not consecutive.

Proof. The proof is immediate from Theorem 2.

\section{Structure of Non-fortuitous States}

The most interesting question that remains is whether such states really exist. Is it possible to get non-fortuitous states of any length $a$ ?

The two examples given by Mantin and Shamir in [1] to establish their claim to the existence of non-fortuitous states need some scrutiny. The first example where $S_{0}[2]=0, i_{0}=0$ and $j_{0}=0$ which gives zero as the second output is claimed to be a 1-predictive 1-state. The claim is not true in the strict sense of the definition (see Def. 2 and [1]) of 1-predictive 1 -state because we impose one more constraint, i.e., $S_{0}[1] \neq 2$, in addition to $S_{0}[2]=0$. However, this inaccuracy is unrelated to the statistical bias in the second output word detected by the authors.

The second example provided in [1] is the RC4 state compatible with $S_{0}[-2]=1, S_{0}[-1]=2, S_{0}[1]=-1, i_{0}=-3$ and $j_{0}=-1 .{ }^{4}$ As the outputs are consecutive, then by Theorem 2 this is a fortuitous state. In Fig. 3 we show that from the $3^{\text {rd }}$ round when $i_{3}=0, j_{3}=3, S_{3}[1]=-1$, $S_{3}[2]=2, S_{3}[3]=1$ the 3-predictive 3-state has become a fortuitous state of length 3 .

\subsection{Determination of Non-fortuitous States: A Step Forward}

Theorem 3. Any 1-predictive 1-state is a fortuitous state of length 1.

Proof. Any 1-predictive 1-state is of the form $i_{0}=2 x-1, j_{0}=x$ and $S_{0}[2 x]=x$. This is clearly a fortuitous state of length 1 ( $x$ is an integer chosen from 0 to $N-1$ ).

\footnotetext{
${ }^{4}$ The symbols $-1,-2,-3$ are used as shorthand for $N-1, N-2, N-3$ respectively throughout this paper.
} 


\begin{tabular}{|c|c|c|c|c|c|c|c|c|c|c|c|}
\hline \multirow[b]{2}{*}{$i$} & \multirow[b]{2}{*}{$j$} & \multicolumn{6}{|c|}{$\mathrm{S}$} & \multirow[b]{2}{*}{$S[i]$} & \multirow[b]{2}{*}{$S[j]$} & \multirow[b]{2}{*}{$S[i]+S[j]$} & \multirow[b]{2}{*}{ Output } \\
\hline & & -2 & -1 & 0 & 1 & 2 & 3 & & & & \\
\hline-3 & -1 & 1 & 2 & $*$ & 1 & $*$ & $*$ & / & / & / & / \\
\hline-2 & 0 & $*$ & 2 & 1 & 1 & * & $*$ & $*$ & 1 & $*$ & $*$ \\
\hline-1 & 2 & $*$ & $*$ & 1 . & 1 & 2 & $*$ & $*$ & 2 & $*$ & $*$ \\
\hline \multicolumn{12}{|c|}{ Fortuitous State } \\
\hline 0 & 3 & $*$ & $*$ & $*$ & 1 & 2 & 1 & $*$ & 1 & $*$ & $*$ \\
\hline 1 & 2 & $*$ & $*$ & $*$ & 2 & -1 & 1 & 2 & -1 & 1 & 2 \\
\hline 2 & 1 & $*$ & $*$ & $*$ & 1 & 2 & 1 & 2 & -1 & 1 & -1 \\
\hline 3 & 2 & $*$ & $*$ & $*$ & 1 & 1 & 2 & 2 & 1 & 3 & 2 \\
\hline
\end{tabular}

Fig. 3. The 3-predictive 3 -state becomes a fortuitous state from the $3^{\text {rd }}$ round.

The above theorem implies that the number of 1-predictive 1-states is $N$ and there is no non-fortuitous state of length 1.

Determination of non-fortuitous states of length $a$, where $a>1$, has inherent difficulties on two counts. Firstly, the relative positions of the SBox elements at the beginning are not known. Secondly, the set of indices containing known elements of the S-Box changes as RC4 runs. The most straightforward and naive method would be to select all possible $a$ indices, assign to the elements pointed to by those indices and $j$ all possible values and finally select those states which generate $a$ non-consecutive outputs (by Corollary 2, non-consecutiveness of outputs is a necessary and sufficient condition for an a-predictive a-state to be non-fortuitous). But the cost of computation, which is $O\left(N^{2 a+1}\right)$ for $a \ll N$, makes such method too costly when $N=256$ and $a=2$ and completely impractical when $a>2$. At this point Finney's forbidden state (see [3]) comes to our rescue. ${ }^{5}$

Proposition 1. The first two elements of the $S$-Box of any a-predictive $a$-state always occupy consecutive places if $a \geq 2$, i.e., $S_{0}[k+1]$ and $S_{0}[k+2]$ (see Fig. 2) always contain known values if $a \geq 2$.

Proof. According to Def. 2, the $1^{\text {st }}$ round always produces output. So, $S_{0}[k+1]$ is occupied with a known element. Assume $S_{0}[k+2]$ is empty. Then $S_{1}[k+2]$ is also empty because any output producing round does not put a known value in any new-cell. In such case $S[i]$ is empty in the $2^{\text {nd }}$ round. Therefore, $j$ cannot be updated and the execution of RC4 stops. Therefore, $S_{0}[k+2]$ is not empty.

Theorem 4. Any 2-predictive 2-state is a fortuitous state of length 2.

\footnotetext{
${ }^{5}$ The cost of computation is measured in terms of the number of value assignments.
} 


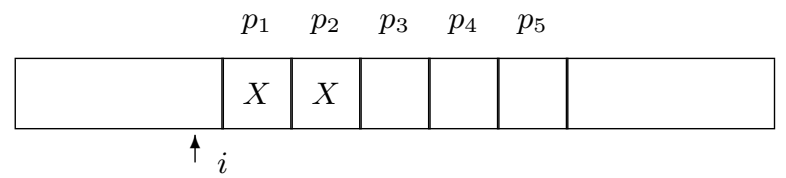

(a) Beginning: $X$ denotes known values.

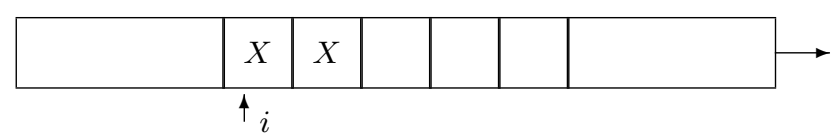

(b) Round 1: When the first output is produced.

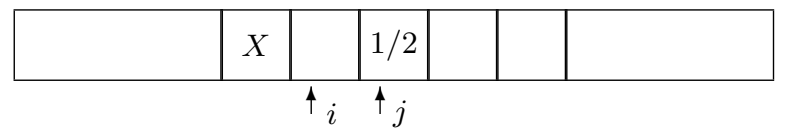

(c) Round 2: No output is produced.

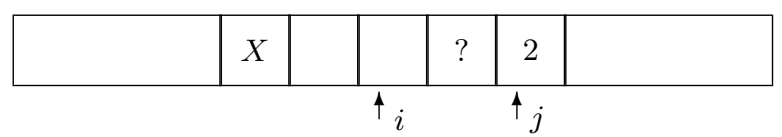

(d) Round 3: No output is produced, $S_{3}\left[p_{4}\right]$ is empty

Fig. 4. Impossibility of getting a non-fortuitous state of length 2. $S_{2}\left[p_{3}\right]=1$ leads to Finney's forbidden state [3]. The $j$ value will be lost from the $4^{t h}$ round. The symbol $1 / 2$ denotes "either 1 or 2 ".

Proof. Let us assume that there is at least one 2-predictive 2-state which is non-fortuitous. Now we try to predict two elements (that must not be consecutive) from this state. From Proposition 1, $S_{0}\left[p_{1}\right]$ and $S_{0}\left[p_{2}\right]$ should contain known elements (see Fig. 4). At round 1 we will have the same positions occupied with known values as round 0 because no new-cell will get any value as output is produced at round 1 . Output cannot be produced at round 2 otherwise we will have two consecutive outputs and clearly that will be a case of a fortuitous state by Theorem 2 . Therefore, at round $2, i$ points to $S_{2}\left[p_{2}\right]$ and $j$ should point to $S_{2}\left[p_{3}\right]$ as shown in Fig. 4(c). For the $j$ value to be available at round $3, S_{1}\left[p_{2}\right]$ should be either 1 (if $j_{1}=p_{2}$ ) or 2 (if $j_{1}=p_{1}$ ). But $S_{1}\left[p_{2}\right]=1$ gives rise to Finney's forbidden state [3], hence it is not possible. The S-Box arrangement at round 2 is shown in Fig. 4(c). So, at round 3, we can not get any output because swapping takes place when $j$ points to an empty cell $S_{3}\left[p_{5}\right]$ (see Fig. 4(d)). After that, $i$ points to $S_{4}\left[p_{4}\right]$ which is still empty. As a consequence, $j_{4}$ cannot be determined and the execution of RC4 halts. Therefore, we can not get any non-fortuitous state of length 2. Thus the theorem is proved. 
Although we are unable to discover any non-fortuitous states so far but with the above results we are confident enough that no such state exists of length 1 or 2 .

\subsection{Determination of Non-fortuitous States: A General Approach}

As mentioned before, two important factors make the determination of non-fortuitous states all the more difficult. Firstly, the relative positions of the $a$ elements at round 0 are not known and secondly, in the subsequent rounds the indices containing the known elements change. Our algorithm is a two-phase one. The first part determines the possible relative positions of the $a$ elements at round 0 . The second part is a state counting algorithm that determines the individual non-fortuitous states. Note, that for a fortuitous state the elements are always consecutive and the set of their indices does not change in the later rounds.

Let $d_{t}$ denote the inter-element gap between the $t^{\text {th }}$ element and the $(t+1)^{t h}$ element. We measure $d_{t}=p_{t+1}-p_{t}-1$ where the $t^{t h}$ element is indexed by $p_{t} .{ }^{6}$ Let $T_{a}$ denote one such sequence $\left(d_{1}, d_{2}, d_{3}, \ldots, d_{a-1}\right)$. Note, that the total number of such sequences is $\sum_{x=0}^{N-a}\left(\begin{array}{c}x+a-2 \\ a-2\end{array}\right)$. So our first step is to sort out the sequences from the exhaustive set; more precisely, we try to reduce the search space. The problem of "relative positions" hinges on an important combinatorial problem: what is the maximum value of $d_{t}$ (we will call this $d_{t}^{\max }$ henceforth) such that, given $\left(d_{1}, d_{2}, d_{3}, \ldots, d_{t-1}\right)$, there exists a sequence $\left(S_{0}\left[p_{1}\right], S_{0}\left[p_{2}\right], \ldots, S_{0}\left[p_{t}\right], j_{0}\right)$ such that $i$ always points to a known value till it reaches the index $p_{t+1}$ ? Proposition 3 of the Appendix A.1 implies that the $i$ pointer has to reach at least $p_{t+1}$ to predict the $(t+1)^{t h}$ output.

There is no known method to determine the exact value of $d_{t}^{\max }$ other than exhaustive search on $t$ elements and $j_{0}$. However, using recursion a loose upper bound, say $\tilde{d}_{t}^{\max }$, can be easily made such that $d_{t}^{\max } \leq \tilde{d}_{t}^{\max }$ and $\tilde{d}_{t}^{\text {max }}$ is much smaller than the trivial maximum value for small values of $a .^{7}$ We will address the problem with respect to non-fortuitous states. The upper bound $\tilde{d}_{t}^{\max }$ will be referred to as $d_{t}^{\max }$ henceforth.

Let $L_{a=t}$ denote the set of all sequences $\left(d_{1}, d_{2}, d_{3}, \ldots, d_{t-1}\right)$ that represent the possible inter-element gaps of the non-fortuitous states of length

\footnotetext{
${ }^{6}$ The $1^{s t}$ element is $S_{0}\left[i_{0}+1\right]$. All known elements in the direction of the movement of $i$ are numbered accordingly.

7 The trivial maximum value of $d_{t}=N-\left(\sum_{1}^{t-1} d_{i}+t+1\right)$.
} 
$t$. Let $d_{t}^{\max }$ correspond to the sequence $\left(d_{1}, d_{2}, d_{3}, \ldots, d_{t-1}\right) \in L_{a=t}$. Then clearly $\left\{\left(d_{1}, d_{2}, d_{3}, \ldots, d_{t-1}, x\right) \mid 0 \leq x \leq d_{t}^{\max }\right\} \subseteq L_{a=t+1}$. Each sequence in $L_{a=t}$ generates a subset and the union of them results in $L_{a=t+1}$. Using Propositions 2 and 3 in Appendix A.1, it can be shown that no sequence of length $(t-1)$ outside $L_{a=t}$ can be a prefix of a sequence in $L_{a=t+1}$.

Now, we outline how $d_{t}^{\max }$ can be evaluated. In fact, we will calculate the maximum value of the index of the $(t+1)^{t h}$ element, say $p_{t+1}^{\max }$. As $d_{t}^{\max }$ does not depend on the values of the indices of the SBox elements, we fix $p_{1}=0$ to ease the computation. We know that $d_{t}^{\max }=p_{t+1}^{\max }-p_{t}-1$. The algorithm is a function Mainfunc which takes a sequence $\left(0, d_{2}, d_{3}, \ldots, d_{t-1}\right)$ as input and calls a recursive function Round in a loop to compute the corresponding $p_{t+1}^{\max }$. Both the functions work 'almost' similarly (the small difference between the functions should not be overlooked). The functions Mainfunc and Round are shown in Appendix A.3 and Fig. 5. $L_{2}, L_{3}, . ., L_{t}$ are all known. Now we describe the recursive function Round. We simulate RC4 without assigning any values to the elements. When $S[i]$ is known which is not assigned a value, the function Maxj computes the maximum value of $j$ (denoted by $j_{\max }$ ) in the current round by matching the sequence of the inter-element gaps of the elements between $i+1$ and $N-1$ with the suitable member from the global lists $L_{2}, L_{3}, . ., L_{t}$. If $j_{\max }$ goes beyond $N-1$ then $j_{\max }=N-1$. The function Range determines the range of $j$ (denoted by $J$ ) such that $i$ may reach up to the location $j$ without pointing to any empty cell in the rounds in between, thereby we reduce the search space. Propositions 2, 3 and 4 in Appendix A.1 establish why it is necessary that $i$ should be able to reach $j$ in the later rounds. The set $J$ includes all the indices containing unknown elements between $i+1$ and $j_{\max }$ excluding those which violate Finney's criterion and uniqueness of permutation elements. $S[i]$ is assigned values such that $j$ takes all possible values within the range. For every value of $S[i]$, the function Round calls itself. In essence, we form a search tree. The function Round returns one of the three types of values. If several branches are originated from the Round function (the case when $S[i]$ is known but not assigned a value before) then the maximum of all the returned values to the current function is stored. It is then compared with $j_{\max }$ at the current round. The greater of the two is returned to the predecessor. ${ }^{8}$ If, at any round function, $S[i]$ is known and already assigned a value then it simply passes on the value from its successor to the predecessor. If, at any round function, $S[i]$ is unknown then

\footnotetext{
${ }^{8}$ Note, if $j$ takes any values outside the range $J$ at some round then $i$ cannot cross $j_{\max }$ in the subsequent rounds and therefore, $j_{\max }$ is returned.
} 
the branch is terminated and $i$ is returned. The branch is also terminated if $i$ sweeps through $N$ indices (in this case $d_{t}^{\max }$ is trivial). The cost of the algorithm is substantially less than the exhaustive search on $t$ elements. Note that, in the Round function, the operation $S^{\prime}=S$ indicates that all elements of the S-box $S$ are copied into a different S-box $S^{\prime}$.

$\operatorname{int} \operatorname{Round}(S, i, j)$

1. $i=i+1$

2. if $i=N-1$ then $\operatorname{return}(i)$

3. if $S[i]$ empty return $(i)$

4. if $S[i]$ known and already assigned a value

1. $j=j+S[i]$

2. $\operatorname{Swap}(S[i], S[j])$

3. $\operatorname{return}(\operatorname{Round}(S, i, j))$

5 . if $S[i]$ known but not assigned any value

1. $j_{\text {max }}=\operatorname{Maxj}(S, i)$

2. $M=0$

3. $J=\operatorname{Range}\left(S, i, j_{\max }\right)$

4. if $J=\phi$ then go to step 6 .

5. For each $x \in J$

1. $S^{\prime}=S, S^{\prime}[i]=x-j$

2. $\operatorname{Swap}\left(S^{\prime}[i], S^{\prime}[x]\right)$

3. $M=\max \left(M, \operatorname{Round}\left(S^{\prime}, i, x\right)\right)$

6. $\operatorname{return}\left(\max \left(M, j_{\max }\right)\right)$

Fig. 5. The round function that computes $p_{t+1}^{\max }$.

We have already observed that, for $a=2, d_{1}=0$ (see Proposition 1). Therefore, $L_{a=2}=\{(0)\}$. We cannot determine $d_{2}^{\max }$ from $L_{a=2}$ as it is constructed following the constraint that the elements are forcefully made consecutive. Applying Finney's forbidden state (described in Sect. 1.2) we determine $d_{2}^{\max }=1$. Therefore, $L_{a=3}=\{(0,0),(0,1)\}$. In the Appendix A.2 we show that $d_{2}^{\max }=1$. Applying the recursion described above we derive $d_{3}^{\max _{1}}=3$ and $d_{3}^{\max _{2}}=3$ that correspond to two different members of $L_{a=3}$. Therefore, $L_{a=4}=\{(0,0,0),(0,0,1),(0,0,2),(0,0,3)$, $(0,1,0),(0,1,1),(0,1,2),(0,1,3)\}$.

Theorem 5. If $p_{t}<N-1$ then $p_{t+1}^{\max }$ returned by Mainfunc is strictly greater than $p_{t} .{ }^{9}$

Proof. A proof is by induction on the length of non-fortuitous states.

To determine the individual non-fortuitous states of length $a=t+1$ by a state counting recursive algorithm (the algorithm works in a similar

${ }^{9}$ Note, by definition $p_{t}>0$. 
manner as the one described before) we take members from $L_{a=t+1}$ one by one, vary $p_{1}$ from 0 to $N-1$ and simulate RC4 without directly assigning values to the elements. In this case, at the first round, $S[j]$ should be one of the known elements. The value of $j_{\max }$ at each of the other rounds is also determined from the lists $L_{2}, L_{3}, . ., L_{t}$. The range of the values of $j$ (other than the first round) is all the indices between $i+1$ and $j_{\max }$ plus the indices containing known values between $p_{1}$ and $i$. That is how the search space is reduced. In this state counting algorithm, using Propositions 3 and 4 in Appendix A.1 it can be shown that if $S[j]$ is known at some round then output has to be produced in that round, i.e., $S[S[i]+S[j]]$ should be a known element as well. This condition effectively reduces the search space again. The algorithm stops whenever $a$ outputs are produced or $S[i]$ is unknown. Among all the states, so obtained, we take only those with non-consecutive outputs.

We computed that the number of non-fortuitous states of length 3 and 4 are 7 and 1727 for $N=256$. In Appendix A.4 we list all the non-fortuitous states of length 3 . It is possible that many members in $L_{a}$ may not eventually produce any non-fortuitous states. But the relative positions of the elements of any non-fortuitous state of length $a$ must correspond to an entry in $L_{a}$. Our attempt is directed to develop a technique to eliminate all the trivial a-states which are impossible to predict $a$ elements.

\section{Cryptanalytic Significance of Non-fortuitous States}

Although we mostly dealt with non-fortuitous predictive states, one can see that the algorithm is more robust, that is, it can as well be used to determine the set of $b$-predictive $a$-states for any $a$ and $b$.

The average number of outputs, needed for any a-predictive a-state to occur, is reduced by knowing the number of non-fortuitous states of length $a$, in addition to that of the fortuitous states of length $a$ [1]. Denote the number of fortuitous states and non-fortuitous states of length a by the symbol $A$ and $B$. Assuming uniformity of the external states and the internal states, a specific elements occur as outputs at specific rounds with probability $N^{-(a+1)}$. The knowledge of non-fortuitous states reduces the length of the output segment required for any a-predictive a-states to happen by $N \cdot N^{a+1}\left(\frac{1}{A}-\frac{1}{A+B}\right)$. Here, the additional factor $N$ comes because of $N-1$ false hits on the average (see Eqn. (1)).

If we use the branch and bound attack [7] on $\mathrm{RC} 4_{n=8}$ with a priori information about 3 elements in the S-Box the attack is not much im- 
proved (an improvement of $\mathbf{2 . 3 6 \%}$ on the number of required output bytes) as we have only 7 non-fortuitous states compared to 290 fortuitous states of the length 3 . If we use a priori information about 4 elements, then with the complete information about non-fortuitous states, we require approximately $2^{34.98}$ output bytes (which is around $\mathbf{2 1 \%}$ less than the earlier estimate of $2^{35.2}$ bytes based on only fortuitous states) for any 4-predictive 4-state to happen. Note, that the number of fortuitous states and non-fortuitous states of length 4 are 6540 and 1727 respectively.

\section{Directions for Future Work and Conclusions}

Our current work leaves room for more research. We proved Corollary 1 with a bound on the number of rounds. For small values of $a$, we observe that the $j$ value is lost much earlier than the $N^{\text {th }}$ round. So, in such cases, Corollary 1 is true even without any bound on the number of rounds. A more challenging combinatorial problem, therefore, is what is the maximum value of $a$ for which Corollary 1 is true without any bound on the number of rounds. From the point of view of cryptanalysis, although we are interested in small values of $a$ (because a small increase in $a$ drastically increases the required outputs for any attack), the problem seems alluring.

Another way to improve the present work is to suggest some elegant algebraic means to determine $d_{t}^{\max }$ when the values of $d_{1}, d_{2}, \ldots, d_{t-1}$ are given: more precisely, one should try to build an algebraic structure for the function $f$ which determines $d_{t}^{\max }$. We see that the set $L_{a}$ contains redundant members which increase the time and space complexity. We are convinced that the algorithm can be further improved.

Our work in this paper is a purely combinatorial analysis of $\mathrm{RC} 4$. We developed a practical scheme to derive a special set of RC4 states known as non-fortuitous predictive states. Apart from that many interesting properties of this cipher (e.g. known $a$ elements cannot predict more than $a$ elements in the next $N$ rounds) are established. We hope these observations will lead to better understanding of the cipher.

\section{Acknowledgements}

We are grateful to Scott Fluhrer for helping us understand the state counting algorithm for fortuitous states. We are thankful to Christophe De Cannière for kindly going through different technical details of the paper and making valuable comments. We also thank Ilya Mironov of Stanford University and Sankardas Roy of George Mason University for useful discussions. 


\section{References}

1. I. Mantin, A. Shamir, "A Practical Attack on Broadcast RC4," Fast Software Encryption 2001 (M. Matsui, ed.), vol. 2355 of LNCS, pp. 152-164, Springer-Verlag, 2001.

2. S. Fluhrer and D. McGrew, "Statiscal Analysis of the Alleged RC4 Keystream Generator," Fast Software Encryption 2000 (B. Schneier, ed.), vol. 1978 of LNCS, pp. 19-30, Springer-Verlag, 2000.

3. H. Finney, "An RC4 cycle that can't happen," Post in sci.crypt, September 1994.

4. I. Mironov, "Not (So) Random Shuffle of RC4," Crypto 2002 (M. Yung, ed.), vol. 2442 of $L N C S$, pp. 304-319, Springer-Verlag, 2002.

5. R. Jenkins, "Isaac and RC4," Published on the Internet at http://burtleburtle.net/bob/rand/isaac.html.

6. J. Golić, "Linear Statistical Weakness of Alleged RC4 Keystream Generator," Eurocrypt '97 (W. Fumy, ed.), vol. 1233 of LNCS, pp. 226-238, Springer-Verlag, 1997.

7. L. Knudsen, W. Meier, B. Preneel, V. Rijmen and S. Verdoolaege, "Analysis Methods for (Alleged) RC4," Asiacrypt '98 (K. Ohta, D. Pei, ed.), vol. 1514 of LNCS, pp. 327-341, Springer-Verlag, 1998.

8. A. Grosul and D. Wallach, "A related key cryptanalysis of RC4," Department of Computer Science, Rice University, Technical Report TR-00-358, June 2000.

9. S. Mister and S. Tavares, "Cryptanalysis of RC4-like Ciphers," SAC '98 (S. Tavares, H. Meijer, ed.), vol. 1556 of LNCS, pp. 131-143, Springer-Verlag, 1999.

10. A. Roos, "Class of weak keys in the RC4 stream cipher," Post in sci.crypt, September 1995.

11. S. Fluhrer, I. Mantin, A. Shamir, "Weaknesses in the Key Scheduling Algorithm of RC4," SAC 2001 (S. Vaudenay, A. Youssef, ed.), vol. 2259 of LNCS, pp. 1-24, Springer-Verlag, 2001.

12. A. Stubblefield, J. Ioannidis and A. Rubin, "Using the Fluhrer, Mantin and Shamir attack to break WEP," NDSS 2002.

\section{A Appendix}

\section{A.1 Criteria for $i$ to reach an index to produce an output}

The fact that the $i$ pointer can move from the index $x$ to the index $y$ implies that the value of $j$ is always available in each of the intermediate $(y-x+1)$ rounds. The S-Box region between the indices $x$ and $y$ is all the $(y-x+1)$ indices from $x$ in the direction of the movement of $i$.

Proposition 2. If, at a particular round $r$ (when $i=i_{r}$ ), $j_{r}$ and some elements of the $S$-Box are known, then the fact that $i$ can reach the index $i_{r}+k$ (where $0<k \leq N$ ) from the round $r$ depends only on $j_{r}$ and the known $S$-Box elements between the indices $i_{r}+1$ and $i_{r}+k$ at round $r$.

Proposition 3. Let the number of known elements of the S-Box at the $r^{\text {th }}$ round between the indices $i_{r}+1$ and $i_{r}+k$ (where $0<k \leq N$ ) be $m$ and at the $r^{\text {th }}$ round the $t^{\text {th }}$ element to the right of $i$ be indexed by $p_{t}$ $(0<t \leq m)$. Then, starting from the $r^{\text {th }}$ round, the pointer $i$ must reach at least $p_{t}$ to predict the $t^{\text {th }}$ output. 
Proposition 4. If the number of rounds, at which $S[j]$ is known during the passage of $i$ from $i=i_{r}$ to $i=i_{r}+k$ (where $0<k \leq N$ ), is $m$, then the number of known elements, between the indices $i_{r}+1$ and $i_{r+k}$ at round $r+k$, is also $m$.

\section{A.2 Evaluation of the maximum value of $d_{2}$}

Theorem 6. For any non-fortuitous state of length $3, p_{3}-p_{2}<3$ where the $t^{\text {th }}$ element is indexed by $p_{t}$.

Proof. Let us assume $p_{3}-p_{2}=3$. Now we try to generate 3 nonconsecutive outputs, in a similar manner as Theorem 4. The execution of the first three rounds are shown in Figure 6 . At the $2^{\text {nd }}$ and the $3^{\text {rd }}$ rounds $j$ must point to $S_{2}\left[g_{1}\right]$ and $S_{3}\left[g_{2}\right]$ respectively, in order for $j$ to be available at the third and the fourth rounds. But such conditions lead to Finney's forbidden state at round three. So our assumption is wrong. Hence, $p_{3}-p_{2} \neq 3$. It is easy to see that the same situation arises for $p_{3}-p_{2}>3$. Therefore, $p_{3}-p_{2}<3$. We know that $d_{2}=p_{3}-p_{2}-1$. Hence, $d_{2}^{\max }=1$.

One can see that if we relax the condition of the $1^{\text {st }}$ round producing output always, then the maximum inter-element gap between the first two elements of the S-Box is also 1. This basic fact will be used in the determination of $d_{t}^{\max }$ when $t>2$.

\section{A.3 The function that calls the Round function}

Mainfunc $\left(\left(0, d_{2}, \ldots, d_{t-1}\right)\right)$

1. Set $p_{1}=0$

2. $i=1$

3. Mark the indices of the S-Box that contain known elements from the sequence $\left(0, d_{2}, \ldots, d_{t-1}\right)$.

4. $j_{\text {max }}=\operatorname{Maxj}(S, i)$

5. $J=$ Range $\left(S, i, j_{\max }\right)$

6. $M=0$

7. For each $x \in J$

1. $S^{\prime}=S, \operatorname{Swap}\left(S^{\prime}[i], S^{\prime}[x]\right)$

2. $M=\max \left(M, \operatorname{Round}\left(S^{\prime}, i, x\right)\right)$

8. $p_{t+1}^{\max }=\max \left(M, j_{\max }\right)$

\section{A.4 List of Non-fortuitous States of length 3}

We list in the form of 5 -tuple $\left(S_{0}[i+1], S_{0}[i+2], S_{0}[i+3], i_{0}, j_{0}\right)$.

1.(0, 1, -2, -3, 0), 2.(0, 1, -1, -3, 0),

3. $(1,3,0,-1,-1)$, 4.(128, 3, 0, -1,-128)

5.(129, 3, 0, -1, -129), 6.(2,-2,0,-1,-1), 7.(2,-1,0,-1,-1) 


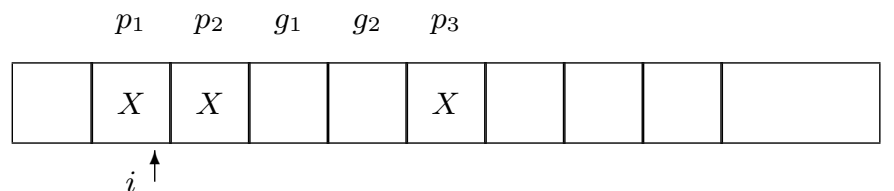

(a)

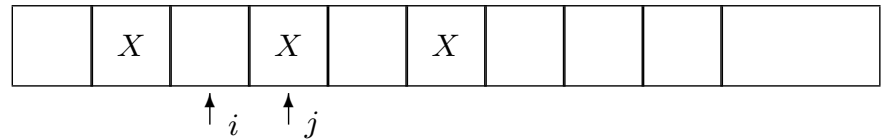

(b)

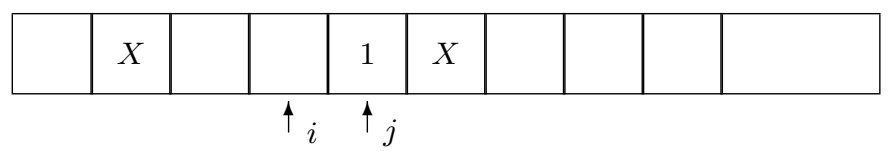

(c)

Fig. 6. A non-fortuitous state of length 3 with $d_{2}=2$. (a) Round 1: After production of the $1^{\text {st }}$ output; $X$ indicates known value. (b) Round 2: No output. (c) Round 3: We reach Finney's forbidden state as $j_{3}=i_{3}+1$ and $S_{3}\left[j_{3}\right]=1$. 